\title{
Prevalencia de herpes, sífilis y condilomatosis en pacientes con VIH en un hospital de referencia de Bogotá
}

Herpes, syphilis and condylomatosis prevalence in HIV patients at a reference hospital in Bogotá

\section{Adriana Mottaㄹ, Diana Patricia Marín², Eliana Andrea Merlo²}

1. Médica dermatóloga, epidemióloga; directora, Posgrado de Dermatología, Universidad El Bosque; jefe, Servicio de Dermatología, Hospital Simón Bolívar, Bogotá, D.C., Colombia.

2. Médica dermatóloga; Universidad El Bosque, Hospital Simón Bolívar, Bogotá, D.C., Colombia.

3. Institución: Hospital Simón Bolívar, Bogotá, D.C., Colombia.

\section{Resumen}

Introducción. La infección por el virus de la inmunodeficiencia humana (VIH) ha afectado a más de 60 millones de personas en el mundo, predisponiéndolas a otras infecciones que aumentan la morbimortalidad. La sífilis, el herpes simple de tipo 2 y los condilomas son coinfecciones frecuentes que modifican el curso del VIH, empeoran el estado de salud de quienes las padecen y conducen a un deterioro rápido que, en ocasiones, puede ser fatal.

Objetivo. Determinar la seroprevalencia de infección por herpes simple de tipo 2 y sífilis, y la prevalencia de condilomatosis en los pacientes con VIH/sida en el Hospital Simón Bolívar de Bogotá en el año 2011.

Métodos. Se llevó a cabo un estudio descriptivo de corte transversal. La muestra estuvo constituida por 220 pacientes positivos para VIH seleccionados de manera consecutiva en algunos servicios de la institución.

Resultados. Se encontró una seroprevalencia de herpes de tipo 2 del 64,9 \% y de sífilis de 43,2 \%. La prevalencia de condilomatosis fue de $36 \%$.

Conclusiones. Existe una alta prevalencia de sífilis, herpes y condilomatosis en pacientes con VIH, por lo cual se hace necesario desarrollar programas de educación sexual enfocados en la prevención de estas infecciones.

PALABRAS CLAVE: sífilis, herpes simple, condiloma acuminado, virus del papiloma humano, enfermedades de transmisión sexual, VIH/sida

\section{Summary}

Introduction: HIV infection has affected over 60 million people worldwide, increasing the risk of other infections that increase morbidity and mortality. Syphilis, herpes simplex type 2 and condylomata are common coinfections that modify the course of HIV, worsen health status of those who suffer it and lead to rapid deterioration that can sometimes be fatal.

Objective: To determine the seroprevalence of herpes simplex type 2 and syphilis, and the prevalence of condilomatosis in patients with HIV/AIDS in the Hospital Simon Bolivar in Bogotá in 2011.

Methods: A cross sectional study was carried out. The sample consisted of 220 HIV positive patients consecutively selected in some departments of the institution.

\section{Correspondencia:}

Eliana Andrea Merlo

Email:

andream244@hotmail.com

Recibido 12 de julio de 2013.

Aceptado: 14 de septiembre de 2013.

No se reportan conflictos de interés. 
Results: Prevalence of type 2 herpes was $64.9 \%$ and syphilis $43.2 \%$. Found condilomatosis prevalence was $36 \%$.

Conclusions: There is a high prevalence of syphilis, herpes and condilomatosis in HIV patients, thus it is necessary to develop sex education programs focused on the prevention of these diseases.

KEY WORDS: Syphilis, herpes simplex, condylomata acuminata, human papillomavirus, sexually transmitted diseases, HIV/AIDS.

\section{Introducción}

Desde su aparición en 1979, la infección por el virus de la inmunodeficiencia humana (VIH) ha afectado a más de 60 millones de personas a nivel mundial y ha llevado a la muerte a más de 20 millones de éstas ${ }^{1,2}$. El primer caso en Colombia se notificó en 1983 y hasta mayo de 2010 se habían registrado 71.526 casos de infecciones por el VIH. La prevalencia en Colombia en la población general es de o,6\%, aproximadamente, aumentando en poblaciones definidas como el grupo de hombres que tienen sexo con hombres, donde existe una prevalencia entre el 10 y el $18 \%{ }^{3}$.

Se reconoce que algunas de las coinfecciones que afectan a estos pacientes varían el curso clínico de la infección, produciendo en ocasiones desenlaces fatales. Entre estas enfermedades hay que mencionar la sífilis, el herpes simple de tipo 2 y la condilomatosis. La sífilis tiene un curso diferente en los pacientes con VIH, evoluciona sin respetar los estadios clásicos conocidos, produce un compromiso neurológico temprano y alteraciones en las pruebas serológicas diagnósticas ${ }^{4-8}$; el herpes simple hace un sinergismo con el VIH cada vez que recidiva, aumentando de esta forma la carga viral del $\mathrm{VIH}^{9}$, y los condilomas aumentan el riesgo de desarrollar neoplasias genitales ${ }^{10}$.

La mayoría de los pacientes con VIH/sida no saben que pueden contagiarse de otras enfermedades de transmisión sexual, no las reconocen clínicamente y desconocen que el adquirirlas puede empeorar su condición inmunológica y producir la muerte ${ }^{11}$. En nuestro país, cuando un paciente con VIH accede a la consulta especializada, en forma rutinaria, se le solicita una prueba serológica para sífilis pero no así para herpes simple; de igual manera, la mayoría de las veces no se realiza una inspección de las regiones anal y genital. Por estas razones, es necesario fomentar acciones preventivas en salud enfocadas a este grupo de pacientes.

Hasta el momento, en Colombia no se había evaluado la prevalencia de estas coinfecciones en pacientes con $\mathrm{VIH} /$ sida, por ello, surgió la iniciativa de realizar un estudio que determinara la seroprevalencia de la infección por herpes simple de tipo 2 y sífilis, y la prevalencia de condilomatosis.

El Hospital Simón Bolívar de Bogotá es un centro de remisión para pacientes con VIH; fue el primero en Bogotá en recibir individuos con esta enfermedad y cuenta con un programa especial de referencia al cual pertenecen 410 pacientes.

El objetivo principal del estudio fue determinar la seroprevalencia de la infección por herpes simple de tipo 2 y sífilis, y la prevalencia de condilomatosis en pacientes con VIH/sida en el Hospital Simón Bolívar de Bogotá, además de describir las características sociodemográficas de la población, cuantificar la carga viral, el recuento de linfocitos T CD4 y describir las asociaciones encontradas entre sífilis, herpes y condilomas según la preferencia sexual.

\section{Materiales y métodos}

Se llevó a cabo un estudio observacional, descriptivo, de corte transversal, con el fin de determinar la seroprevalencia de herpes simple de tipo 2 y sífilis, y la prevalencia de condilomatosis, en pacientes infectados con VIH/sida atendidos en el Hospital Simón Bolívar. Para esto se tomó como población blanco los pacientes con infección por $\mathrm{VIH} /$ sida que consultaron al Hospital Simón Bolívar que, para el año 2011, eran 410.

Previa aprobación del Comité de Ética del Hospital Simón Bolívar, se incluyeron pacientes con infección por VIH/sida que tuvieran diagnóstico confirmado de VIH mediante una prueba de ELISA y Western Blot. Se excluyeron pacientes con discapacidad cognitiva o alteración del estado de conciencia. El tamaño de la muestra se calculó mediante la fórmula de Hosmer-Lemeshow, y se incluyeron 222 pacientes que asistían a consulta progra- 


\section{"La mayoría de los pacientes con VIH/sida no saben} que pueden contagiarse de otras enfermedades de

\section{transmisión sexual, no las reconocen clínicamente} y desconocen que el adquirirlas puede empeorar su

\section{condición inmunológica y producir la muerte"}

mada de VIH, que estaban hospitalizados, en urgencias o que asistieron al laboratorio clínico en agosto de 2011. Cada paciente fue incluido en el estudio, previo diligenciamiento del consentimiento informado.

Se realizó una encuesta de 77 preguntas, un examen físico para evaluar clínicamente la presencia de condilomas y se tomó la muestra de sangre respectiva, todo en el mismo momento. En la encuesta se incluyeron 7 preguntas sobre variables sociodemográficas, 7 sobre variables relacionadas con los antecedentes de sexualidad del paciente, 7 sobre VIH/sida, 6 sobre el abuso de sustancias lícitas e ilícitas, 12 para conocer el estadio B del VIH, 26 para determinar el estadio C, 2 para evaluar el reconocimiento del estado de infección por el virus del herpes simple de tipo 2, 1 de reconocimiento de la infección por sífilis, 3 de reconocimiento de condilomas genitales y 6 con los resultados de las pruebas de laboratorio.

En el panel de exámenes se solicitó reagina plasmática rápida cuantitativa, la cual se realizó mediante la detección de anticuerpos contra componentes de Treponema pallidum, con el suero en diferentes diluciones ( $\mathrm{o}$, 1:2, 1:4, 1:8 y mayor de 1:16). La prueba se realiza en el suero humano que puede contener anticuerpos contra $T$. pallidum, se investiga con un antígeno artificial, dando aglutinación en caso positivo y luego con el suero a diluciones dobles progresivas en solución salina. Se solicitó absorción de anticuerpos fluorescentes del treponema (FTA-ABS) mediante la prueba Sero-Bacter USR (Cat. 01.00.1, Laboratorios Biobacter). Los resultados fueron reportados como reactivos, limítrofes (borderline) y no reactivos. Finalmente, se detrminó la inmunoglobulina $\mathrm{G}$ (IgG) para el virus herpes simple de tipo 2 mediante una prueba inmunoenzimática indirecta que detecta anticuerpos específicos de tipo IgG frente a herpes simple de tipo 2 en suero humano mediante el método de unión antígeno-anticuerpo (type-specific glycoprotein G, gG2) del Laboratorio Vircell.

Se definió como caso de seroprevalencia para sífilis al paciente con un título reactivo de FTA-ABS, como caso posible de sífilis activa al paciente con un título de reagina plasmática rápida positivo mayor de 1: 160 más, y caso de seroprevalencia positiva para infección por herpes simple de tipo 2 el paciente con un título de IgG por inmunoensayo enzimático para herpes simple igual o mayor de 12 unidades. Los resultados menores de 9 fueron interpretados como negativos, y de 9 a 11, dudosos. El caso de condilomatosis se definió por la presencia de condliomas en el momento del examen. Asimismo, se realizó una revisión de la historia clínica de cada uno de los pacientes.

El procesamiento de los datos se hizo con el programa SPSS, versión 15.0. Se determinaron los porcentajes y las frecuencias para las variables cualitativas y medidas de tendencia central y dispersión para las variables cuantitativas. Se estableció la seroprevalencia de herpes simple de tipo 2 y sífilis y la prevalencia de condilomatosis en pacientes con infección por VIH/sida. Se calculó el odds ratio (OR) para la asociación entre la preferencia sexual y la presencia de infecciones de transmisión sexual, y asociación entre ser trabajador sexual y las infecciones de transmisión sexual evaluadas en el presente estudio.

\section{Resultados}

El $82 \%$ de los pacientes del estudio eran hombres con una edad promedio de 39 años, la mayoría se encontraban solteros (64\%). El 8o \% de los pacientes pertenecía a estratos socioeconómicos del 1 al 3. El $35 \%$ de la muestra había tenido relaciones sexuales con personas de su mismo sexo. El 90,1 \% de los pacientes no usaba 
Característica

n

SEXo

Masculino

Femenino

ESTADO CIVIL

Soltero

Unión libre

Casado

Separado

Viudo

EDAD

Rango:17 a 69 años

Promedio: 38,7 años

Desviación estándar: 10.9

Nivel de EDUCACIón

Secundaria

Primaria

Profesional

Técnico

Sin educación

ESTRATO SOCIOECoNómico

Estrato 3

Estrato 2

Estrato 1

Estrato 5

Desplazados

Indigentes

Cárcel

Estrato 6

TRABAJADORES SEXUALES

Sí

No

Preferencia Sexual

$\begin{array}{lcc}\text { Heterosexuales } & 103 & 46,6 \% \\ \text { Homosexuales } & 78 & 35,3 \% \\ \text { Bisexuales } & 40 & 18,1 \%\end{array}$

MÁs DE UNA PAREJA EN LOS ÚlTIMOS 6 MESES

Sí $\quad 36 \quad 16.6 \%$

EDAD DE LA PRIMERA RELACIÓN SEXUAL

Mínima: 5; máxima: 30

NÚMERO DE PAREJAS SEXUALES

Mínimo: 1; máximo: 500

Promedio: 42,7

Desviación estándar: 78

USO DE PRESERVATIVO ANTES DE ADQUIRIR

LA INFECCIÓN CON VIH

No $200 \quad 90 \%$

USO DE PRESERVATIVO DESPUÉS DE ADQUIRIR

LA INFECCIÓN CON VIH

Sí $151 \quad 68 \%$

TERAPIA HAART

Sí

RECUENTO DE LINFOCITOS T CD4

Entre 200 y 498

CARGA VIRAL MENOR DE 100 COPIAS

ESTADIO B DEL VIH

ESTADIO C DEL VIH

$200 \quad 90,1 \%$

$51 \%$

$47 \%$

$18 \%$

$74,8 \%$

TABLA 1. Características de los pacientes del estudio. preservativo antes de adquirir la infección con VIH, después de adquirirla lo usaba el $68 \%$. Respecto al inicio en la vida sexual, el promedio de la población tuvo su primera relación a los 15 años de edad, y la cuarta parte antes de los 14 años de edad, tratándose de niños que fueron abusados sexualmente.

Solamente fue posible obtener los datos de linfocitos CD4 y cargas virales en 200 de los pacientes. De la totalidad de los pacientes, $47 \%$ tenía carga viral menor de 100 copias. Cerca de la mitad de los pacientes, presentaba recuento de linfocitos T CD4 entre 200 y 498 (TABLA 1).

$\mathrm{Al}$ estudiar los pacientes con estadio $\mathrm{B}$, gran parte presentó fiebre o diarrea por más de dos semanas, al igual que candidiasis oral y herpes zoster, siendo estas tres infecciones las más frecuentes en este grupo de pacientes. La mayoría de los pacientes $(74,8 \%)$ se encontraba en estadio $\mathrm{C}$ y, de ellos, la mitad presentó desgaste y pérdida de peso, seguido por herpes simple y neumonía por Pneumocistis jiroveci.

El $84 \%$ de los pacientes se encontraba recibiendo terapia antirretroviral. En cuanto a pacientes seropositivos para sífilis, se encontraron 77 (35\%), de los cuales, siete $(3,2 \%)$ presentaban diluciones de $1: 8$ y diecinueve $(8,6 \%)$ reagina plasmática rápida mayor de $1: 16$, los cuales se consideraron como casos de sífilis activa y se incluyeron en un programa de tratamiento. El FTA-ABS fue reactivo en 96 pacientes ( $43 \%$ ), de los cuales, solo 66 pacientes $(29,7 \%)$ conocían que habían presentado sífilis en algún momento de su vida. La serología IgG para herpes simple de tipo 2 fue positiva (mayor de 11) en 144 de los pacientes $(64,9 \%)$. De los 144 pacientes con serología positiva para herpes de tipo 2, 29 (20 \%) refirieron haber presentado úlceras y vesículas genitales, 12 (8\%) solo úlceras y 13 (9\%) vesículas en la región genital; 90 pacientes $(63 \%)$ no habían presentado sintomatología asociada al herpes de tipo 2. La presencia de condilomatosis se evidenció en 30 pacientes (13,5\%). La localización de los condilomas se distribuyó así: 24 (30 $\%)$ en la región genital, 43 (56 \%) en la región perianal y 11 (14\%) con localización en ambos sitios. Los hombres fueron los afectados en mayor proporción (95\%). El antecedente de condilomatosis fue referido por 80 pacientes (36\%) (TABLA 2).

El carcinoma de cuello uterino se encontró en cuatro de las mujeres del estudio, uno de estos casos asociado al antecedente de condilomatosis. El antecedente de displasia cervical fue comentado por cinco de las pacientes, tres de ellas habían sido tratadas, además, por condilomatosis genital.

Se encontró asociación entre ser trabajador sexual y la presencia de sífilis $(\mathrm{OR}=3,5)$ y ser trabajador sexual con la presencia de condilomatosis $(\mathrm{OR}=3,62)$. El tener relaciones con personas del mismo sexo se encontró 


$\begin{array}{lll}\text { Resultado } & \mathbf{n} & \mathbf{( \% )} \\ \text { RPR reactivo } & 77 & 35 \\ \text { RPR reactiva en 1:2 } & 41 & 18,5 \\ \text { RPR reactiva en 1:4 } & 10 & 4,5 \\ \text { RPR reactiva en 1:8 } & 7 & 3,2 \\ \text { RPR reactiva en 1:16 } & 19 & 8,6 \\ \text { FTA-ABS reactivo } & 96 & 43 \\ \text { Serología IgG para herpes } & & \\ \text { simple, tipo 2 } & & \\ \text { Positiva } & 144 & 64,9 \\ \text { Sin lesiones } & 90 & 63 \\ \text { Úlceras genitales } & 12 & 8 \\ \text { Vesículas genitales } & 13 & 9 \\ \text { Úlceras y vesículas genitales } & 29 & 20 \\ \text { Antecedente de condilomas } & 80 & 36 \\ \text { Presencia de condilomas } & 30 & 13,5 \\ \text { Región genital } & 24 & 30 \\ \text { Región perianal } & 43 & 56 \\ \text { Región genital y perianal } & 11 & 14\end{array}$

TABLA 2. Resultados pruebas serológicas en el examen físico.

asociado a la presencia de sífilis $(\mathrm{OR}=6,13)$, herpes $(\mathrm{OR}=3,57)$ y condilomas ( $\mathrm{OR}=2,75)$; mientras que ser bisexual se asociaba con la presencia de sífilis $(\mathrm{OR}=4,7)$

(TABLA 3).

\section{Discusión}

Este estudio muestra la importancia de realizar una búsqueda activa de otras infecciones de transmisión sexual en la población de pacientes con VIH.

La seroprevalencia de sífilis en la población estudiada, medida como la reactividad en la prueba FTAABS fue de 43,2 \%, más alta con respecto a los estudios realizados con poblaciones similares, como el de Badie, et al., en Teherán donde se reportó una seroprevalencia de $0,45 \%{ }^{12}$, o el de Adias en España que evidenció una seroprevalencia del 29,8\% $\%^{13}$. En Madrid en un centro de referencia de pacientes con VIH se encontró una seroprevalencia de $7,1 \%{ }^{14}$, y en Israel en un grupo de militares, de 5,8\% $0^{15}$.

En el Hospital Simón Bolívar se encontró una asociación entre tener relaciones con personas del mismo sexo y la presencia de sífilis $\left(\mathrm{OR}=6,13\right.$; $\left.\mathrm{IC}_{95 \%}: 2,97-12,65\right)$, asociación también descrita por Ortega en Madrid $(\mathrm{OR}=32,6$; $\left.\mathrm{IC}_{95 \%}: 16,2-65,4\right)(14)$. A pesar de que el diseño de este estudio fue transversal, es importante mencionar que estos pacientes requieren un seguimiento serológico periódico, pues los trabajos que así lo hacen demuestran una incidencia anual del $1,3 \%{ }^{15}$.

Para observar qué conciencia de haber padecido sífilis, tenían los pacientes con FTA-ABS reactivo, se obtuvo el indice kappa entre la variable FTA-ABS y una variable que cuestionaba si los pacientes conocían que habían tenido sífilis; el valor de kappa fue de o,54 con una p de o, lo que implica que no hay una buena concordancia. Teniendo en cuenta que el índice kappa es una medida de correlación o concordancia, si es menor de o no hay ninguna concordancia; entre o y o,2 es insignificante; entre 0,2 y 0,4, baja; entre 0,4 y 0,6, moderada; entre o,6 y o,8, buena, y entre 0,8 y 1, muy buena. Un alto porcentaje de los pacientes con VIH infectados por sífilis son asintomáticos por lo que carecen de conciencia de la enfermedad ${ }^{16}$.

La seroprevalencia de herpes simple de tipo 2 fue de $64,9 \%$, comparable con los diferentes datos reportados

\begin{tabular}{|c|c|c|c|c|c|c|c|c|c|}
\hline & \multicolumn{3}{|c|}{ Herpes } & \multicolumn{3}{|c|}{ Sífilis (FTA-ABS) } & \multicolumn{3}{|c|}{ Condilomas } \\
\hline & OR & IC & $\mathrm{p}$ & OR & IC & $\mathrm{p}$ & OR & IC & $\mathrm{P}$ \\
\hline Heterosexual & 1 & . & . & 1 & . & . & 1 & . & . \\
\hline Homosexual & 3,57 & $(1,77-7,20)$ & 0,0001 & 6,13 & $(2,97-12,65)$ & 0,0000 & 2,75 & $(1,42-5,31)$ & 0,0016 \\
\hline Bisexual & 1,51 & $(0,71-3,21)$ & 0,2786 & 4,7 & $(2,02-11)$ & 0,0001 & 2,69 & $(1,21-5,95)$ & 0,0108 \\
\hline $\begin{array}{l}\text { Trabajador } \\
\text { sexual }\end{array}$ & 3,6 & $(0,77-16,74)$ & 0,0806 & 3,5 & $(1,04-11,71)$ & 0,0302 & 3,62 & $(1,15-11,42)$ & 0,0185 \\
\hline Herpes & & & & 2,19 & $(1,21-3,95)$ & 0,0075 & 1,31 & $(0,73-2,35)$ & 0,3638 \\
\hline Sífilis & 2,19 & $(1,21-3,95)$ & 0,0075 & & & & 1,49 & $(0,87-2,62)$ & 0,8476 \\
\hline Condilomas & 1,31 & $(0,73-2,35)$ & 0,3638 & 1,49 & $(0,87-2,62)$ & 0,1628 & & & \\
\hline
\end{tabular}

TABLA 3. Asociaciones. 
en la literatura, como lo muestra un estudio australiano realizado por Russell en el que la seroprevalencia fue de $66,7 \%{ }^{17}$, o un estudio en Zimbabue que reportó una seroprevalencia de 79,2 \% en trabajadores sexuales ${ }^{18}$, o en Morelos (México) donde se encontró un $48,1 \%{ }^{19}$; asimismo, fue diferente de la encontrada por Janbakhash en Irán donde la cifra fue del $6,5 \%{ }^{20}$.

La prevalencia de condilomatosis en el presente estudio fue del $36 \%$, comparable con estudios previos. La localización más frecuente fue la región perianal, similar a lo encontrado en varios estudios, en los que se muestra que un gran porcentaje de pacientes con VIH son hombres que tienen sexo con hombres. En Nicaragua se encontró una prevalencia del $46 \%$, y la localización más frecuente fue la región perianal ${ }^{21}$. En Medellín, entre 349 pacientes con VIH, 37,8 \% presentaron condilomatosis, y en el $42 \%$ fueron de localización anal ${ }^{22}$.

En la población estudiada encontramos una asociación entre tener relaciones con personas del mismo sexo y la presencia de condilomas (OR=2,75; $\left.\mathrm{IC}_{95 \%} 1,42-5,31\right)$, dato similar al del estudio de Darwich $\left(\mathrm{OR}=2,2\right.$; $\mathrm{IC}_{95 \%}$ 1,4-3,5) ${ }^{23}$.

El carcinoma de cuello uterino encontrado en 4 de las mujeres del estudio, se asoció al antecedente de condilomatosis, y de los cinco casos de mujeres con displasia cervical, tres habían padecido condilomas, lo cual puede estar relacionado con el potencial oncogénico que en estas pacientes tiene el virus del papiploma humano.

$\mathrm{Al}$ cruzar las preferencias sexuales con la presencia de infecciones de transmisión sexual, el tener relaciones con personas del mismo sexo genera un riesgo de entre 2,7 a 6,3 veces de adquirir condilomas, herpes y sífilis comparado con ser heterosexual. Ser bisexual aumenta el riesgo entre 1,5 a 4,7 veces de tener sífilis, herpes y condilomas. Al evaluar la condición de ser o no trabajador sexual y la presencia de infecciones de transmisión sexual se encontró que hay un riesgo entre 3,5 a 3,6 veces de tener sífilis, herpes y condilomas.

La presencia simultánea de las tres infecciones de transmisión sexual se encontró en 33 pacientes $(14,8 \%)$, tan sólo tres de estos heterosexuales, 10 bisexuales y 20 personas que tienen sexo con otras de su mismo sexo.

\section{Conclusiones}

Existe un alto porcentaje de pacientes con VIH/sida y enfermedades de transmisión sexual, lo cual obliga en forma urgente a proponer un programa educativo dirigido a la prevención de infecciones de transmisión sexual y al desarrollo de programas de detección temprana, diagnóstico y tratamiento oportuno, considerando las sinergias entre el VIH, el herpes, la sífilis y la presencia de condilomatosis que provocan un empeoramiento del VIH/sida.

\section{Agradecimientos.}

A Daniel Toledo, Lili Rueda y Alexandra Rojas.

\section{Referencias}

1. Joint United Nations Program on HA. 2004 Report on the global AIDS epidemic 4th global report. Geneva: UNAIDS; 2004.

2. OMS. Situación de la epidemia del SIDA: Informe especial sobre la prevención del VIH. Ginebra: ONUSIDA; 2007.

3. Moreno T. Casos de VIH/sida en Colombia notificados al SIVIGILA, 2008. Inf Quinc Epidemiol Nac. 2009;14:97-107.

4. Simms I, Fenton KA, Ashton M, Turner KM, Crawley-Boevey $\mathrm{EE}$, Gorton $\mathrm{R}$, et al. The re-emergence of syphilis in the United Kingdom: The new epidemic phases. Sex Transm Dis. 2005;32:220-6.

5. Fenton KA, Imrie J. Increasing rates of sexually transmitted diseases in homosexual men in Western Europe and the United States: Why? Infect Dis Clin North Am. 2005;19:311-31.

6. Rompalo AM, Lawlor J, Seaman P, Quinn TC, Zenilman JM, Hook EW, 3rd. Modification of syphilitic genital ulcer manifestations by coexistent HIV infection. Sex Transm Dis. 2001;28:448-54.

7. Hutchinson CM, Hook EW, 3rd, Shepherd M, Verley J, Rompalo AM. Altered clinical presentation of early syphilis in patients with human immunodeficiency virus infection. Ann Intern Med. 1994;121:94-100.

8. D’Amico R, Zalusky R. A case of lues maligna in a patient with acquired immunodeficiency syndrome (AIDS). Scand J Infect Dis. 2005;37:697-700.

9. Lama JR, Lucchetti A, Suárez L, Laguna-Torres VA, Guanira JV, Pun $\mathrm{M}$, et al. Association of herpes simplex virus type 2 infection and syphilis with human immunodeficiency virus infection among men who have sex with men in Perú. J Infect Dis. 2006;194:1459-66.

10. Lee PK, Wilkins KB. Condyloma and other infections including human immunodeficiency virus. Surg Clin North Am. 2010;90:99-112.

11. Díez M, Díaz A. Sexually transmitted infections: Epidemiology and control. Rev Esp Sanid Penit. 2011;13:58-66.

12. Badie, Yavari Z, Esmaeeli S. Prevalence survey of infection with Treponema pallidum among HIV-positive patients in Tehran. Asian Pac J Trop Biomed. 2013;3:334-6.

13. Díaz A, Junquera ML, Esteban V, Martínez B, Pueyo I, Suárez J, et al. HIV/STI co-infection among men who have sex with men in Spain. Euro Surveill. 2009 Dec 3; 14(48). pii: 19426

14. Pulido F, Rubio R, Salmerón OJ,Reactivity of serologic tests for the detection of syphilis in patients infected with the human immunodeficiency virus. Med Clin (Barc). 1993;101:365-7.

15. Ganesan A, Fieberg A, Agan BK. Results of a 25-year longitudinal analysis of the serologic incidence of syphilis in a cohort of HIVinfected patients with unrestricted access to care. Sex Transm Dis. 2012;39:440-8.

16. Branger J, van der Meer JT, van Ketel RJ, Jurriaans S, Prins JM.High incidence of asymptomatic syphilis in HIV-infected MSM justifies routine screening. Sex Transm Dis. 2009;36:84-5. 
17. Russell DB, Tabrizi SN, Russell JM, Garland SM. Seroprevalence of herpes simplex virus types 1 and 2 in HIV-infected and uninfected homosexual men in a primary care setting. J Clin Virol. 2001;22:305-13.

18. Cowan FF, Pascoe SJ, Barlow KL, Langhaug LF, Jaffar S, Hargrove JW, Robinson NJ, Latif AS, Bassett MT, Wilson D, Brown DW, Hayes RJ.Interaction of HSV-2 and HIV in rural Zimbabwean sex workers (SW). AIDS. 2006;20:261-7.

19. Yáñez I, Martínez M, Conde C, García A, Sánchez M. Seroprevalencia y seroincidencia del virus del herpes simple tipo 2. Enf Inf Microbiol. 2011;31;93-7.

20. Janbakhash A, Mansouri F, Vaziri S, Sayad B, Afsharian M, Abedanpor A.Seroepidemiology of herpes simplex virus type 2 (HSV2) in HIV infected patients in Kermanshah-Iran. Caspian J Intern Med. 2012;3:546-9.
21. Encuesta Centroamericana de Vigilancia de Comportamiento Sexual y Prevalencia de VIH e ITS en Poblaciones Vulnerables en Nicaragua - ECVC. ONUSIDA, Nicaragua 2011.

22. Gaviria M, Orozco B, Gómez L, Maya C, Estrada S, Peláez L, Cataño, J. Manifestaciones dermatológicas en pacientes con VIH: seguimiento en 349 pacientes. Rev Asoc Colomb Dermatol. 2007;15-18

23. Darwich L, Cañadas MP. Condylomata, cytological abnormalities and human papillomavirus infection in the anal canal in HIVinfected men. HIV Med. 2012;13:549-57. 